\title{
MODE-SPECIFICITY IN PHOTODISSOCIATION OF FUNDAMENTAL STRETCHINGS OF HOD
}

\author{
I. BAR, Y. COHEN, D. DAVID, T. ARUSI-PARPAR, S. ROSENWAKS and J.J. VALENTINI* \\ Department of Physics, Ben-Gurion University of the Negev, IL-Beer-Sheva 84105, Israel \\ ${ }^{*}$ Department of Chemistry, Columbia University, New York, NY 10027, USA
}

\begin{abstract}
Stimulated Raman excitation and coherent anti-Stokes Raman scattering prepare and detect, respectively, HOD molecules with one quantum of vibrational excitation in the O-H or O-D stretch vibrations. An ArF excimer laser at $193 \mathrm{~nm}$ promotes vibrationally excited molecules to the first electronic surface $A\left({ }^{1} B_{1}\right)$ where they dissociate to produce $\mathrm{OH}+\mathrm{D}$ or $\mathrm{OD}+\mathrm{H}$ fragments. The photodissociation products $\mathrm{OD}$ and $\mathrm{OH}$ are detected via laser induced fluorescence. The photodissociation cross section of HOD $(0,0,1)$ vibrationally excited molecules with one quantum of vibration in the $\mathrm{O}-\mathrm{H}$ stretch is greatly enhanced over that for $\operatorname{HOD}(0,0,0)$. The dissociation of this vibrationally excited molecule is also very selective, the $O D+H$ yield being $2.5 \pm 0.5$ times greater than the $\mathrm{OH}+\mathrm{D}$ yield. In the photodissociation of the O-D stretch, excited HOD $(1,0,0)$, no enhancement of the yield of the fragments is obtained. Also, these results demonstrate that bond cleavage does not necessarily occur on the weakened bond, and they agree with theoretical calculations indicating that the yield of $\mathrm{OD}$ and $\mathrm{OH}$ fragments depends on the Franck-Condon overlap of the ground state vibrational wavefunction with the continuum wavefunction on the repulsive surface of the upper state. Our results show that even the very lowest possible level of vibrational excitation can be "leveraged" to effect selective bond breaking.
\end{abstract}

\section{1.- Introduction.}

Mode-selective control of the reaction pathway by selective excitation of particular reagent vibrational states which correspond to specific motions in the molecule is of much interest in unimolecular reactions. The initiation of a bondselected chemical reaction can be achieved by special energy investment in the reaction coordinate, either by its direct excitation or by preferential coupling of energy into it from another vibration $\left({ }^{1}\right)$. Selective excitation of the parent molecule with one quantum of vibrational excitation is the cleanest possible way to get such specific energy deposition in a particular bond, since coupling to other vibrational modes is minimized for such excitation and often even specific rotational levels can be selectively pumped. This energy can then induce the molecule to dissociate selectively along the vibrationally excited coordinate in a photodissociation process.

The HOD molecule is particularly interesting for investigating the influence of reagent fundamental vibrational excitation in bond-selective photodissociation, since theoretical predictions on this molecule are available. The first fully three-dimensional quantum evaluation of mode-selective bond fissions has been carried out by Segev and Shapiro (2) for HOD in the second absorption band $(B \leftarrow X)$ and predicted enhanced yields for the $\mathrm{OH}+\mathrm{D}$ channel. Studies on HOD photodissociation conducted by Engel and Schinke $(3)$ and independently by Imre and coworkers $\left({ }^{4}\right)$ using ab initio potential energy surfaces $\left(^{5}\right)$ indicate that this molecule potentially can also exhibit mode-specific behavior in the first absorption band $(A \leftarrow X)$. These authors have predicted the isotopic branching ratio for the photodissociation $H O D \rightarrow O D+H(O H+D)$, and showed that the ratio is a strong function of the frequency of the dissociating photon and the vibrational state of the HOD. The theoretical studies predict $O D / O H$ ratios that agree with the results for photodissociation of $\operatorname{HOD}(4 \mathrm{VOH})\left({ }^{6}\right)$, for $157 \mathrm{~nm}$ photolysis of $\operatorname{HOD}(0,0,0)(7)$, and with our results for $193 \mathrm{~nm}$ photodissociation of $\operatorname{HOD}(0,0,1)(8)$. Similarly, good agreement with theory $(9,10)$ is obtained for the $\mathrm{OH}$ product quantum state distribution in 157 and $193 \mathrm{~nm}$ photodissociation of $\mathrm{H}_{2} \mathrm{O}(0,0,0)$ and in $193 \mathrm{~nm}$ photolysis of $\mathrm{H}_{2} \mathrm{O}(0,0,1)$ prepared by infrared excitation $(9,10,11)$. 
The major motivation of this study is to control the reaction dynamics of molecules from particular rovibrational states, which are selected in the vibrational excitation step. The most important aspect of this is the preparation of different, distinct initial vibrational states by selective investment of minimal amounts of energy. This paper describes the photodissociation dynamics of HOD excited with one quantum of vibrational energy in the pure $\mathrm{O}-\mathrm{H}$ or $\mathrm{O}-\mathrm{D}$ stretches. The selective excitation of HOD with just one quantum of vibrational excitation in the O-H or O-D stretches leads to different photodissociation channels. When HOD $(0,0,1)$ molecules are photodissociated the $\mathrm{OH}+\mathrm{D}$ and especially the $\mathrm{OD}+\mathrm{H}$ yields are appreciably enhanced relative to $\mathrm{HOD}(0,0,0)$, while when $\mathrm{HOD}$ $(1,0,0)$ molecules are photodissociated no enhancement of the yields of either $\mathrm{OH}+\mathrm{D}$ or $\mathrm{OD}+\mathrm{H}$ is observed. These experiments that prepare and subsequently photodissociate a particular low lying initial state provide an important test of theoretical predictions $(3,4)$.

\section{2.- Experiment.}

The experimental protocol involves excitation of a specific fundamental vibrational mode of HOD, photolysis of the vibrationally excited molecule, and laser induced fluorescence (LIF) (12) detection of the hydroxyl species $\mathrm{OH}$ and OD. Vibrational excitation of $\operatorname{HOD}(0,0,1)$ and $\operatorname{HOD}(1,0,0)$ is effected by stimulated Raman excitation (SRE), and the excited molecules are monitored by coherent anti-Stokes Raman scattering (CARS) $\left({ }^{13}\right)$.

In the SRE technique the frequency difference of two visible lasers is tuned to the transition of a specific fundamental vibrational mode. Here we use the second harmonic of a Nd:YAG laser at $532 \mathrm{~nm}\left(\omega_{\mathrm{p}}\right)$ and a tunable dye laser $\left(\omega_{s}\right)$ pumped by it for the two visible beams. Excitation of the molecule is monitored and optimized by measuring the CARS signal $\left(\omega_{a s}\right)$ generated by the Raman excitation beams. Following the SRE pulse, after a delay of $50 \mathrm{~ns}$, the vibrationally excited molecules are photodissociated by a $193 \mathrm{~nm}$ beam $\left(\omega_{\mathrm{d}}\right)$ from an ArF excimer laser. After a delay of another $50 \mathrm{~ns}$ the $\mathrm{OH}$ and $\mathrm{OD}$ photofragments are probed by LIF on the $\mathrm{A}, \mathrm{v}^{\prime}=0 \leftarrow \mathrm{X}, \mathrm{v}^{\prime \prime}=0$ transition, with $\sim 307 \mathrm{~nm}$ pulse $\left(\omega_{\mathrm{pr}}\right)$ from the frequency-doubled output of a Nd:YAG-pumped dye laser.

The $\omega_{\mathrm{p}}, \omega_{\mathrm{s}}$ and $\omega_{\mathrm{pr}}$ beams propagate collinearly through the cell, after passing through a lens, which brings them to a common focus at the center of the cell. At the exit of the gas cell $\omega_{\mathrm{p}}, \omega_{\mathrm{s}}, \omega_{\mathrm{pr}}$ and $\omega_{\mathrm{as}}$ are transmitted by a dichroic mirror that reflects $\omega_{\mathrm{d}}$. The beams are recollimated by a lens, then $\omega_{\mathrm{as}}$ is separated from the input beams by a PellinBroca prism and color filters and focused onto a slit in front of a photomultiplier tube (PMT). The photodissociation beam $\omega_{\mathrm{d}}$ counterpropagates, relative to the SRE and LIF beams, through a lens which brings it to the focus of the other beams. The LIF from the hydroxyl fragments is observed at a right angle to the laser beams and is imaged by a 2" focal length $\mathrm{f} / 1$ lens onto a $5 \mathrm{~mm}$ slit in front of a PMT and an interference filter $(310 \pm 5 \mathrm{~nm})$. A boxcar integrator (SRS, SR250 and SR280) captures the resulting LIF and CARS signals. A PC controls the scanning of the dye laser. It also accumulates, pulse by pulse, the LIF and CARS signals along with signals from photodiodes that monitor the energy of the photodissociation and probe lasers. The LIF is normalized to the photolysis and probe laser energies. Since the CAR.S signal is saturated with respect to each of the exciting beams the LIF signal is not normalized to it. The experin $n$ ts are carried out in a gas cell through which the sample flows at a pressure of 0.35 Torr. The sample is drawn from a liquid reservoir containing a statistical mixture of $\mathrm{HOD}, \mathrm{H}_{2} \mathrm{O}$ and $\mathrm{D}_{2} \mathrm{O}$ with $\mathrm{H}: \mathrm{D}$ ratio of 1 . Because of the differences in the vibrational frequencies of $\mathrm{HOD}, \mathrm{H}_{2} \mathrm{O}$ and $\mathrm{D}_{2} \mathrm{O}\left({ }^{14}\right)$, the SRE produces no vibrationally excited $\mathrm{H}_{2} \mathrm{O}$ or $\mathrm{D}_{2} \mathrm{O}$ when tuned for HOD excitation.

\section{3.- Results.}

Shown in panel (a) of figure 1A is the CARS spectrum obtained by tuning the SRE excitation through the Q-branch transitions of $(0,0,1)$. Panels $(b)$ and $(c)$ present the $O D\left(v^{\prime \prime}=0\right)$ and $O H\left(v^{\prime \prime}=0\right)$ LIF signals as a function of the SRE scan with the probe laser fixed on the $\mathrm{R}_{2}(4)$ transitions for $\mathrm{OD}$ and $\mathrm{OH}$, respectively. The position of the rotational lines in panels (b) and (c) correspond to those seen in the CARS spectrum (a). These peaks are due to the photodissociation of molecules in particular rotational states of the $\mathrm{O}-\mathrm{H}$ stretch vibiation. It is clearly seen that the $\mathrm{OH}$ and OD photofragment yields are enhanced when the $(0,0,1)$ state is prepared by SRE. Comparison of the OD fluorescence to that of $\mathrm{OH}$ demonstrates that the photodissociation of $\mathrm{HOD}(0,0,1)$ always produces more OD than $\mathrm{OH}$ and the branching ratio of the $\mathrm{OD} / \mathrm{OH}$ product, is $2.5 \pm 0.5$. The top panel of figure 1B illustrates the CARS signal obtained by tuning the SRE excitation through the Q-branch transitions of $(1,0,0)$. Panels (b) and (c) show the $R_{2}(4)$ LIF signals of the $O D$ and $O H$ photofragments when the SRE is tuned through rotational levels of $(1,0,0)$, the $\mathrm{O}-\mathrm{D}$ stretch excitation in HOD. No enhancement of the $\mathrm{OH}$ and $\mathrm{OD}$ fluorescence is observed, in contrast to the strong enhancement of $\mathrm{OD}$ and $\mathrm{OH}$ yields for photodissociation of $\mathrm{HOD}(0,0,1)$. 


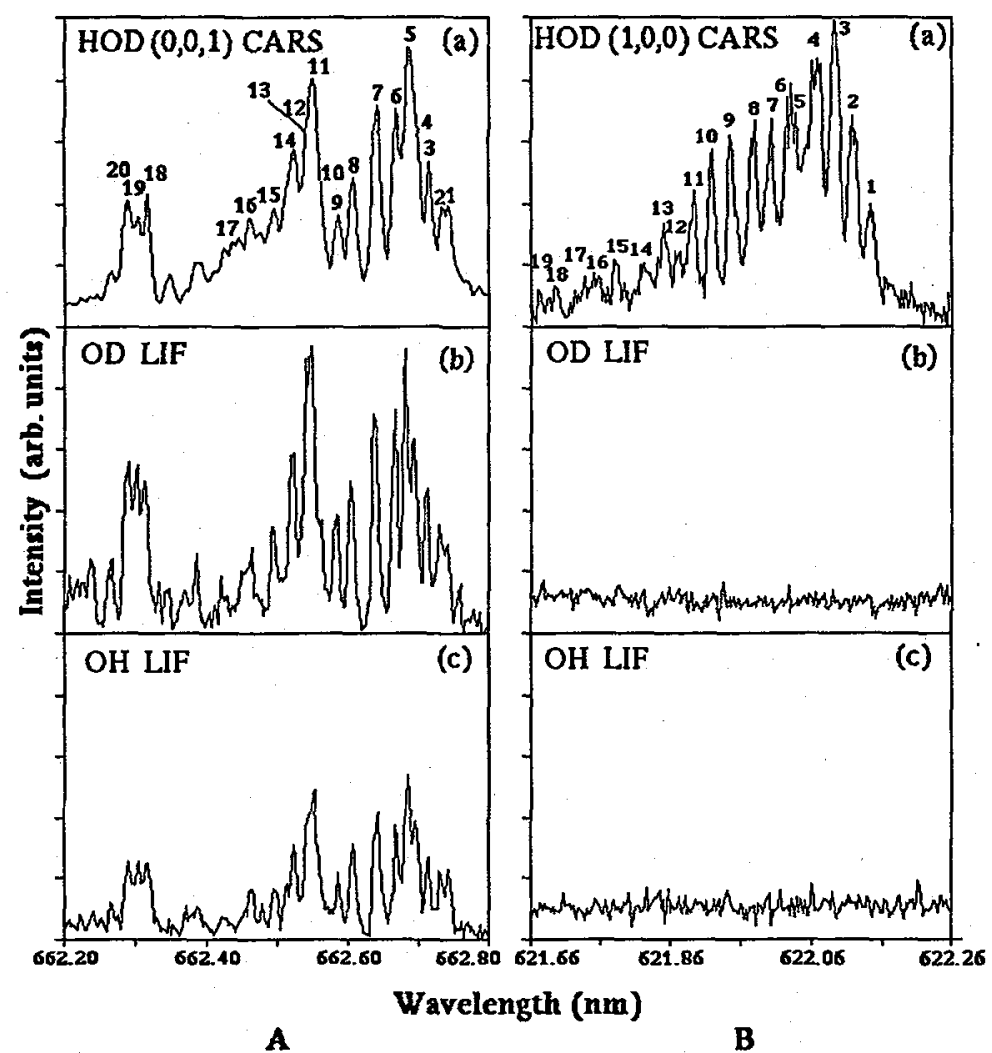

Fig. 1 Direct excitation (A) of HOD $(0,0,1)$ and (B) of HOD $(1,0,0)$ obtained by scanning the SRE excitation through the Q-branch rotational transitions of the vibrational states: (a) saturated CARS signal, (b) and (c) LIF of the $\mathrm{R}_{2}$ (4) transition of the OD and $\mathrm{OH}$ fragments, respectively. The vertical scale in (b) and (c) is the same. The background signal due to photodissociation of vibrationless ground state molecules, which is comparable to the signal resulting from photodissociation of $\operatorname{HOD}(0,0,1)$ molecules, has been subtracted.

\section{4.- Discussion and Summary.}

Our measurements show that the lowest possible level of vibrational excitation of HOD has a dramatic effect on the photodissociation cross section, and that the effect is vibrational-mode-specific. These results also indicate that the vibrational excitation leads to bond selective dissociation. The enhancement of the photodissociation cross section is very large. Extrapolation of the measured $\left({ }^{15,16}\right)$ water absorption band leads to an estimate of $5 \times 10^{-22} \mathrm{~cm}^{2}$ for the absorption cross section of the $(0,0,0)$ vibrational state water molecules at $193 \mathrm{~nm}$. We estimate that $30 \%$ of the HOD molecules having Raman transitions within the bandwidth of the SRE lasers are excited to $(0,0,1)$. The Boltzmann distribution over rotational states determines the percentage of the molecules having Raman transitions within the SRE bandwidth. On an average for our experimental conditions $\sim 2 \%$ of the HOD molecules fall within the SRE bandwidth. Therefore, about $0.6 \%$ of the HOD molecules ( $0.3 \%$ of all the water isotopomers) are vibrationally excited. Relying on the experimental results we estimate that the yield of OD from $H O D(0,0,1)$ is about two times larger than that from the $(0,0,0)$ isotopomers. This implies that at $193 \mathrm{~nm}$ the photodissociation cross section for HOD $(0,0,1)$ is $~ 300$ times larger than that of the $(0,0,0)$ molecules.

In contrast to the behavior of $\operatorname{HOD}(0,0,1)$, for $\operatorname{HOD}(1,0,0)$ the theoretical work predicts no enhancement of the photodissociation cross section. Our results are consistent with this prediction; as shown in Fig. 1 we see no increase in the yield of $\mathrm{OH}$ or OD fragments when we prepare $\mathrm{HOD}(1,0,0)$. Based on our signal-to-noise ratio we estimate that the $193 \mathrm{~nm}$ photodissociation cross section for $\operatorname{HOD}(1,0,0)$ is no more than six times that of the $(0,0,0)$ molecules. Thus, the cross section for $\operatorname{HOD}(0,0,1)$ at $193 \mathrm{~nm}$ is at least 50 times greater than that of $H O D(1,0,0)$, even though these two states differ in energy by only about $2 \%$ of the bond energy.

The magnitude of the bond selectivity we observe in the fragmentation of the HOD $(0,0,1)$ is also consistent with the theoretical predictions of Imre and co-workers $\left({ }^{4}\right)$, at least qualitatively. We find that breaking the $\mathrm{O}-\mathrm{H}$ bond to form $\mathrm{OD}+\mathrm{H}$ is favored over breaking the $\mathrm{O}-\mathrm{D}$ bond to form $\mathrm{OH}+\mathrm{D}$ by a factor of $2.5 \pm 0.5$. The theoretically predicted 
ratio, $\sim 10$, is actually considerably greater than we measure. However, the predicted branching ratio is estimated from the thoretical $O H+D$ and $O D+H$ partial absorption cross section for $H O D(0,0,1)$, and since the absorption cross section for the $\mathrm{OH}+\mathrm{D}$ channel is very small the estimate is only approximate.

The theoretical calculations reveal that Franck-Condon (FC) effects are the source of both the difference in photodissociation behavior of $\operatorname{HOD}(0,0,1)$ and $\operatorname{HOD}(1,0,0)$ and the selectivity in the bond breaking when HOD $(0,0,1)$ is dissociated. The enhancement of the photodissociation cross section of HOD $(0,0,1)$ is the result of a much better FC overlap of the dissociative continuum with the $(0,0,1)$ vibrational wave function than with either the $(0,0,0)$ or the $(1,0,0)$ vibrational wave functions. Even though the vibrational excitation of the O-D stretch in HOD $(1,0,0)$ gives the O-D bond an initial "push" in the direction of the dissociation coordinate to $\mathrm{OH}+\mathrm{D}$, the unfavorable FC factor keeps this from enhancing the dissociation. In the case of HOD $(0,0,1)$ the favorable FC factor enables the additional energy along the $\mathrm{OD}+\mathrm{H}$ dissociation coordinate to be very effective at enhancing the dissociation.

Comparison of the $193 \mathrm{~nm}$ photodissociation of $\operatorname{HOD}(0,0,1)$ and $\operatorname{HOD}(1,0,0)$ molecules to the vibrationally mediated photodissociation of HOD $(4 \mathrm{vOH})$ at $239.5 \mathrm{~nm}$ shows that although the excess energy for photodissociation over bond dissociation energy are comparable $\left({ }^{17}\right)$, the relative yields of the hydroxyl photofragments are different, $\mathrm{OD} / \mathrm{OH} \sim 2.5, \mathrm{OD} / \mathrm{OH} \sim 1$ and $\mathrm{OD} / \mathrm{OH}>15$, respectively. In the experiments of Crim and coworkers $\left({ }^{6}\right)$ the vibrational excitation to $\mathrm{v}=4$ is quite high, about $1.7 \mathrm{eV}$ or $33 \%$ of the $5.118 \mathrm{eV}$ HOD bond dissociation energy, and about $24 \%$ of the $6.9 \mathrm{eV}$ energy of the photons that effect dissociation. However, in our experiments the vibrational excitation energy is very small, only about $9 \%$ and $7 \%$ of the dissociation energy and only about $7 \%$ and $5 \%$ of the energy of the photons that effect dissociation, for HOD $(0,0,1)$ and HOD $(1,0,0)$, respectively. These results and the studies of single photon photodissociation of $\operatorname{HOD}(0,0,0)$ at $157 \mathrm{~nm}(7)$ support theoretical calculations $(3,4)$. The present study, where two distinct vibrational modes are prepared before the photodissociation, confirms that it is not enough to weaken the bond to be cleaved in order to get bond selectivity, but a good FC overlap of the vibrational wave function with the corresponding channel on the dissociative excited state is essential.

To conclude, the photodissociation of HOD prepared in particular rovibratational states in the fundamental vibrational stretching modes, photodissociated with a $193 \mathrm{~nm}$ beam and detected by LIF of the $\mathrm{OH}$ and $\mathrm{OD}$ photofragments, has been investigated. The most striking result of these experiments is the observation that it is possible to control the branching ratio of the $\mathrm{OH}$ and $\mathrm{OD}$ fragments by specific selection of the fundamental vibrational wavefunctions. Excitation of the $\mathrm{O}-\mathrm{H}$ stretching, $\operatorname{HOD}(0,0,1)$, leads to a preference in the $\mathrm{O}-\mathrm{H}$ bond fission, while that of O-D stretching, HOD $(1,0,0)$, does not enhance the O-D bond cleavage. Our results demonstrate that bond cleavage does not necessarily occur on the weakened bond, but rather depends on the FC overlap between the initial and final states. This agrees with theoretical studies and shows that even at the very lowest level of vibrational excitation, selective bond breaking can be effected.

Acknowledgement. This work has been supported by a grant from the United States-Israel Binational Science Foundation (BSF), Jerusalem, Israel. JJV acknowledges support of this work from the National Science Foundation.

5.- References.

/1) F.F. Crim, Science 249, 1387 (1990).

RI E. Segev and M. Shapiro, J. Chem. Phys. 77, 5604 (1982).

B/ V. Engel and R. Schinke, J. Chem. Phys. 88, 6831, (1988).

14/ D.G. Imre and J. Zhang, Chem. Phys. 139, 89 (1989).

15/ V. Staemmler and A. Palma, Chem. Phys. 93, 63 (1985).

16/ R.L. Vander Wal, J.L. Scott and F.F. Crim, J. Chem. Phys. 92, 803, (1990).

II N. Shafer, S. Satyapal and R. Bersohn, J. Chem. Phys. 90, 6807, (1989).

18/ I. Bar, Y. Cohen, D. David, T. Arusi-Parpar, S. Rosenwaks and J.J. Valentini, J. Chem. Phys., in press;

I. Bar, Y. Cohen, D. David, S. Rosenwaks and J.J. Valentini, J. Chem. Phys. 93, 2146, (1990).

19/ K. Weide, S. Hennig and R. Schinke, J. Chem. Phys. 91, 7630, (1989).

110/ P. Andresen and R. Schinke, in Molecular Photodissociation Dynamics, edited by M.N.R. Ashfold and J.E. Baggott (Royal Society of Chemistry, London, 1987) p. 61.

111 A.U. Grunewald, K.H. Gericke and F.J. Comes, Chem. Phys. Lett. 133, 501 (1987).

112/ J.L. Kinsey, Annu. Rev. Phys. Chem. 28, 349 (1977).

/13/ J.J. Valentini, in Laser Spectroscopy and its Applications, Opt. Eng., Vol. 11, edited by L.J.

Radziemsky, R.W. Solarz and J.A. Paisner (Marcel Dekker, New York, 1987) p. 507.

/14/ W.S. Benedict, N. Gailar and E.K. Plyler J. Chem. Phys. 24, 1139 (1956).

/15/ K. Watanabe and M. Zelikoff, J. Opt. Soc. Am. 43, 753 (1953).

/16/ A.H. Laufer and J.R. McNesby, Can. J. Chem. 43, 3487 (1965).

/17/ The excess energy for photodissociation of $\operatorname{HOD}(0,0,1)$ and $\operatorname{HOD}(1,0,0)$ at $193 \mathrm{~nm}$ is $14236 \mathrm{~cm}^{-1}$, and $13252 \mathrm{~cm}^{-1}$, respectively, and for photodissociation of HOD ( $4 \mathrm{vOH}$ ) at $239.5 \mathrm{~nm}$ is $14310 \mathrm{~cm}^{-1}$. 